\title{
Análisis de falla operacional de un sistema hidroneumático en instalación hospitalaria
}

\section{Operational failure analysis of a hydro- pneumatic system in a hospital facility}

DOI: http://dx.doi.org/10.17981/ingecuc.14.1.2018.14

Artículo de investigación científica. Fecha de recepción: 05/01/2018. Fecha de aceptación: 15/06/2018

José P. Monteagudo Yanes

Universidad de Cienfuegos. Cienfuegos (Cuba)

jpmyanes@ucf.edu.cu

Reinier Jiménez Borges

Universidad de Cienfuegos. Cienfuegos (Cuba)

rjborges@ucf.edu.cu

Para citar este artículo:

R. Jiménez Borges y J. P. Monteagudo Yanes, "Análisis de falla operacional de un sistema hidroneumático en instalación hospitalaria", INGE CUC, vol. 14, no. 1, pp. 151-158, 2018. DOI: http://doi.org/10.17981/ingecuc.14.1.2018.14

\section{Resumen}

Introducción- El trabajo presenta el estudio de la falla operacional de un sistema hidroneumático destinado al suministro de agua a una institución hospitalaria.

Objetivo- Determinar la o las causas de interrupción del suministro de agua del sistema hidroneumático a la edificación.

Metodología-Se trazó la característica hidráulica del sistema de tubería y se comparó con la característica hidráulica del sistema hidroneumático, comprobando que satisfacía plenamente las solicitudes de carga y flujo requeridas por la edificación. Dada la presencia de ruidos y vibraciones en la tubería de succión se realizó la comparación del NPSH requerido con el NPSH disponible, determinando que el actual diseño de la tubería de succión es la causa de la falla del sistema hidroneumático.

Resultados-Se proponen tres variantes de modificación al diseño de la tubería de succión que eliminan el fenómeno de la cavitación. Ello evita poner en operación una bombea auxiliar que incrementa el consumo energético en $8760 \mathrm{kWh} /$ año con un costo de 1839,6 \$/año y la inseguridad en el servicio de agua.

Conclusiones- El equipo hidropresor seleccionado cumple satisfactoriamente los requerimientos de la instalación, exceptuando el diseño de la tubería de succión donde el NPSH disponible es inferior al NPSH requerido para caudales superiores a $900 \mathrm{l} / \mathrm{min}$, apareciendo el fenómeno de la cavitación y provocando la salida de servicio del sistema.

Palabras clave- NPSHd, NPSHr, cavitación, bombeo, carga, succión

\begin{abstract}
Introduction- This paper presents the study of the operational failure of a hydro-pneumatic system for the supply of water to a hospital facility.

Objective- Determine the cause or causes for the interruption of the water supply of the hydro-pneumatic system to the building.

Methodology-The hydraulic characteristic of the pipe system was drawn and compared with the hydraulic characteristic of the hydro-pneumatic system, verifying that it fully satisfied the load and flow requests demanded by the building. Given the presence of noises and vibrations in the suction pipe, the comparison of the required NPSH vs. the available NPSH determined that the current design of the suction pipe is the cause for the hydro-pneumatic system failure.

Results- Three variants for the modification of the design of the suction pipe in order to eliminate the phenomenon of cavitation are proposed. This avoids putting into operation an auxiliary pump that increases energy consumption by $8,760 \mathrm{kWh} /$ year with a cost of $1,839.6$ $\$ / y e a r$ and the insecurity in the water service.

Conclusions- The selected hydro-pneumatic equipment satisfactorily meets the requirements of the installation, except for the design of the suction pipe where the available NPSH is lower than the NPSH required at flow rates higher than $900 \mathrm{l} / \mathrm{min}$, hence, producing the cavitation phenomenon and causing the output of system's service.
\end{abstract}

Keywords- NPSHd, NPSHr, cavitation, pumping, head, suction 


\section{INTRODUCCIÓN}

Uno de los tipos de bombas más utilizados a nivel industrial son las bombas centrífugas. Ellas representan cerca del $22 \%$ del consumo nacional de energía eléctrica de países desarrollados y el $18 \%$ en el consumo industrial [1].

La adecuada selección e instalación del equipo de bombeo se inicia por el diseño del sistema de tubería; una correcta correspondencia entre el equipo de bombeo y el sistema de tubería garantizará un funcionamiento estable y seguro del equipo de bombeo y evitará la presencia de la cavitación [2]. El conocimiento e identificación de las variables que propician dicho fenómeno físico permitirá preservar el equipo operando por periodos prolongados de vida útil [3].

En los líquidos es posible obtener una presión muy baja debido al movimiento del fluido, y si ésta disminuye hasta la presión de vapor del líquido a la temperatura dada, entonces se presenta la ebullición [2] y el fenómeno de cavitación. En el estudio propuesto por [3] se presentan algunas de las variables que favorecen la cavitación, siendo estas, la presión atmosférica del lugar en donde está instalada la bomba; la presión de vapor del líquido bombeado; la altura de succión de la bomba al eje del impulsor; y las pérdidas en la succión [4-5].

Cualquier discusión de las limitaciones de presión en el lado de la succión requiere de la comprensión del término Carga Neta Positiva en la Succión (NPSH) [6-10]. Además, existen dos términos de carga positiva en la succión, la Carga Neta Positiva en la Succión requerida $\left(\mathrm{NPSH}_{\mathrm{R}}\right)$, la cual requiere el equipo de bombeo para prevenir la cavitación y es usualmente suministrado por el fabricante, y la Carga Neta Positiva en la Succión disponible $\left(\mathrm{NPSH}_{\mathrm{d}}\right)$ la cual depende fundamentalmente de las condiciones de operación y de la geometría del sistema de conducción. A bajos valores de flujo, el NPSHR se encuentra entre 1,5-2,5 m y se incrementa con aumentos de flujo [11]. Generalmente, los datos de NPSHR se suministran para una velocidad específica y diámetro de impelente. Para propósitos de diseño, puede ser añadido como nivel de seguridad un intervalo entre 0,5-1,0 m [11]. El trabajo de [12] propone un método de optimización multiobjetivo para la familia de bombas centrífugas de doble succión, donde a partir de un modelo experimental aproxima el valor del NPSHR, siendo el valor predictivo de $3,89 \mathrm{~m}$ y el valor de prueba de 4,07 m. Por otra parte, [13] en su estudio valora la medición del rendimiento de una bomba para varias condiciones de operación con el propósito de obtener las curvas características, así como de analizar el NPSHR para diferentes flujos. [14] enfoca su estudio en una evaluación experimental del rendimiento de una bomba y del fenómeno de la cavitación. Las pruebas de cavitación, de acuerdo con [14], revelaron que, para cargas parciales, el NPSHd fue de 90,9 kPa y, para carga total, fue de $129,4 \mathrm{kPa}$, siendo necesaria para garantizar la estabilidad de la operación.
El objetivo de la presente investigación es comprobar si la causa de la salida de servicio del sistema hidroneumático se debe a la no correspondencia del equipo seleccionado con el sistema de tubería en que debe trabajar o a la presencia de cavitación en la instalación.

\section{Metodología}

La falla del sistema hidroneumático reportado por la dirección de la institución se presenta en el régimen de operación establecido. Después de la puesta en marcha, la máquina opera con los parámetros establecidos por un período cercano a las dos horas; pasado este tiempo se observa una reducción en el suministro de agua y, a posteriori, se inician ruidos ligeros y vibraciones hasta que cesa el bombeo de líquido.

Para garantizar el suministro de líquido a la institución, la dirección de mantenimiento aprobó la puesta en marcha del sistema sustituido, consistente en dos bombas centrífugas conectadas en paralelo, sin embargo, el mal estado técnico de las mismas pone en peligro la continuidad de los servicios hospitalarios a falta del suministro de agua.

A solicitud de la dirección de mantenimiento de la institución hospitalaria, se inicia el estudio del nuevo sistema instalado. En la Fig. 2 se ofrece un esquema simplificado del mismo y los parámetros básicos del sistema, el cual está compuesto por tres bombas del fabricante Sea Land modelo CN50-200C [16] y el hidropresor perteneciente a la misma firma, modelo AV800. La Fig. 1 presenta una vista de los equipos de la instalación.

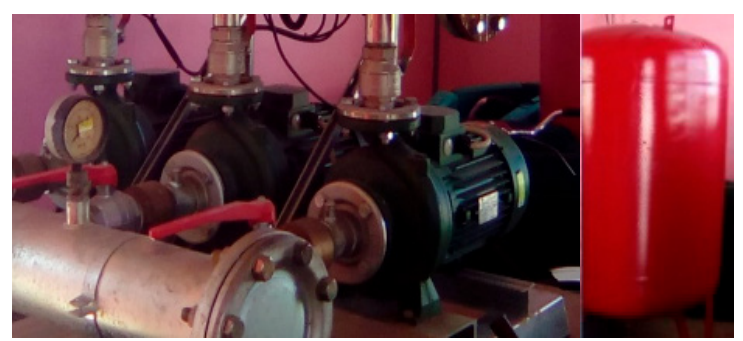

Fig. 1. Sistema de bombas centrífugas e hidropresor Sea Land. Fuente: Autores.

En la Fig. 2 se ofrece un esquema simplificado del mismo y los parámetros básicos del sistema.

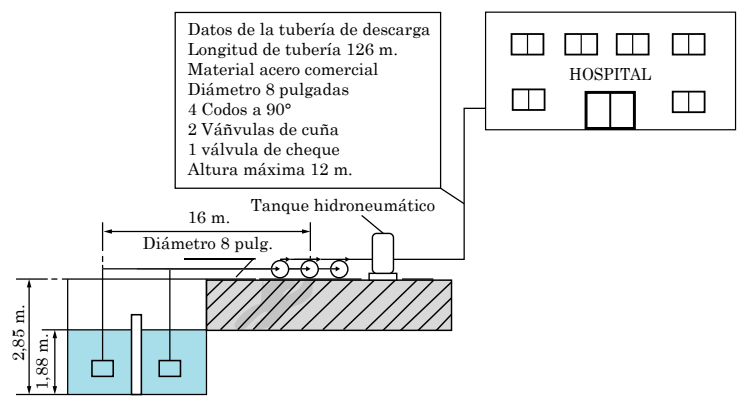

Fig. 2. Esquema simplificado del sistema de tubería con el sistema hidroneumático. Fuente: Autores. 
Aplicando la ecuación de Bernoulli (ecuación 1) entre las regiones de succión y descarga sobre las superficies de los niveles de líquido, se determina la carga de impulsión que deben vencer los equipos de bombeo conectados al tanque del sistema hidroneumático.

$$
\begin{aligned}
H_{b} & =\frac{P_{2}-P_{1}}{\rho * g}+\frac{V_{2}^{2}-V_{1}^{2}}{2 * g}+\Delta Z \\
& +\left[\lambda * \frac{L}{d} * \frac{Q^{2}}{2 * g * A^{2}}+\frac{Q^{2}}{2 * g * A^{2}} * \sum_{i=1}^{n} K_{i}\right]
\end{aligned}
$$

Donde:

$P n$ : Presión en la superficie del líquido en los tanques de succión y descarga $(\mathrm{N} / \mathrm{m} 2)$

$V n$ : Velocidad de la superficie del líquido en los tanques de succión y descarga $(\mathrm{m} / \mathrm{s})$

$\Delta Z$ : Diferencia de altura entre los niveles de los tanques de succión y descarga (m)

$\rho$ : Densidad del fluido $(\mathrm{kg} / \mathrm{m} 3)$

g: Aceleración gravitatoria $(\mathrm{m} / \mathrm{s} 2)$

$\lambda$ : Coeficiente de fricción en el tramo recto de tubería (adimensional)

L: Longitud del tramo recto de tubería (m)

$d$ : Diámetro de la tubería (m)

$Q$ : Caudal de fluido trasegado por la tubería (m3/s)

$A$ : Área de la sección transversal de la tubería (m2)

$K$ : Coeficiente del accesorio (adimensional)

$i$ : Número del accesorio

$n$ : Número del accesorio final

En la tabla 1, se muestra la solución de la ecuación 1 a partir de los parámetros de funcionamiento, conociendo que la institución requiere un caudal de fluido entre 800 y 1000 l/min

Tabla 1. Cálculo de la característica HIDRÁULICA DEL SISTEMA DE TUBERÍA.

\begin{tabular}{|c|c|c|c|c|c|}
\hline $\begin{array}{c}\text { Caudal } \\
(\mathrm{Q})\end{array}$ & $\mathrm{Q}^{2}$ & $\begin{array}{c}\lambda^{*} \mathrm{~L} / \mathrm{d}^{*} \\
\left(\mathrm{Q}^{\wedge} 2 / 2^{*} \mathrm{~g}^{*} \mathrm{~A}^{2}\right)\end{array}$ & $\mathrm{KQ}^{2}$ & $\Delta \mathrm{Z}$ & $\begin{array}{c}\text { Hsist } \\
\text { máx }\end{array}$ \\
\hline$(\mathrm{l} / \mathrm{min})$ & $\left(\mathrm{m}^{6} / \mathrm{s}^{2}\right)$ & $(\mathrm{m})$ & $(\mathrm{m})$ & $(\mathrm{m})$ & $(\mathrm{m})$ \\
\hline 0 & 0 & 0 & 0 & 14 & 14 \\
\hline 200 & 0,000011 & $8,63 \mathrm{E}-08$ & 0,31 & 14 & 14,31 \\
\hline 300 & 0,000025 & $1,94 \mathrm{E}-07$ & 0,70 & 14 & 14,70 \\
\hline 400 & 0,000044 & $3,45 \mathrm{E}-07$ & 1,25 & 14 & 15,25 \\
\hline 500 & 0,000069 & $5,39 \mathrm{E}-07$ & 1,96 & 14 & 15,96 \\
\hline 600 & 0,000100 & $7,76 \mathrm{E}-07$ & 2,82 & 14 & 16,82 \\
\hline 700 & 0,000136 & $1,05 \mathrm{E}-06$ & 3,84 & 14 & 17,84 \\
\hline 800 & 0,000178 & $1,38 \mathrm{E}-06$ & 5,01 & 14 & 19,01 \\
\hline 900 & 0,000225 & $1,74 \mathrm{E}-06$ & 6,34 & 14 & 20,34 \\
\hline 1000 & 0,000278 & $2,15 \mathrm{E}-06$ & 7,83 & 14 & 21,83 \\
\hline 1100 & 0,000336 & $2,61 \mathrm{E}-06$ & 9,47 & 14 & 23,47 \\
\hline 1200 & 0,000400 & $3,10 \mathrm{E}-06$ & 11,27 & 14 & 25,27 \\
\hline
\end{tabular}

Fuente: Autores.

En la Fig. 3, se muestra la representación gráfica de la característica hidráulica del sistema de tubería.
Para comprobar la pertinencia de la selección del equipo en operación, se traza la característica hidráulica del sistema de tubería (Fig. 3), sobre la característica hidráulica dada por el fabricante (Fig. 4).

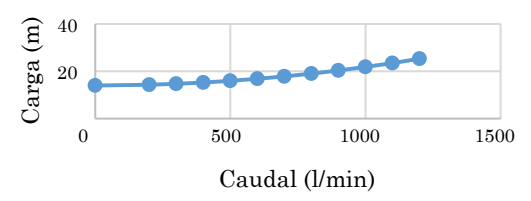

Fig. 3. Característica hidráulica del sistema de tubería. Fuente: Autores.

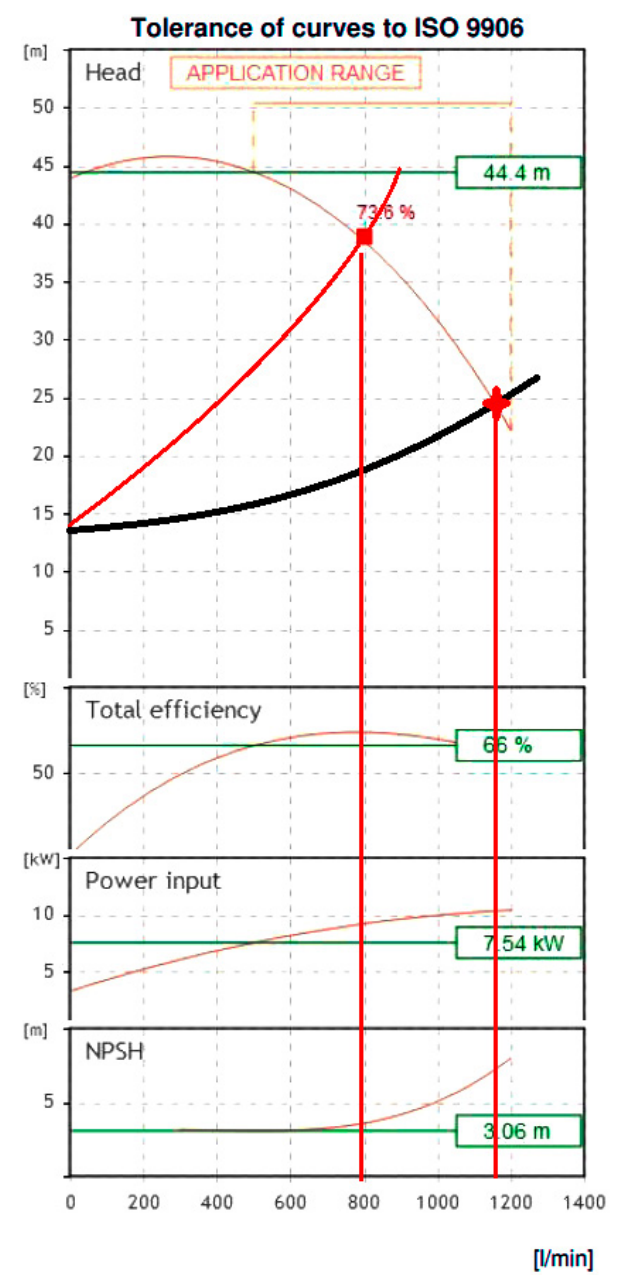

Fig. 4. Trabajo conjunto del sistema de tubería con las características hidráulicas de las máquinas. Fuente: SEA LAND Italia. Modelo CN 50 - 200 C.

En la Fig. 4 se demuestra que la selección del equipo hidroneumático es adecuada para el sistema de tubería de la instalación hospitalaria. A plena capacidad, la máquina entrega un caudal cercano a los $1150 \mathrm{l} / \mathrm{min}$ venciendo una carga de $25 \mathrm{~m}$, con un consumo de potencia en el orden de $11 \mathrm{~kW}$, un rendimiento del $66 \%$ y con un NPSH requerido de $8 \mathrm{~m}$, aproximadamente. 
Dado que el caudal solicitado por la entidad es de $8001 / \mathrm{min}$, es necesario efectuar un estrangulamiento en la válvula de suministro al hospital y ello lleva a la máquina a un estado de funcionamiento de $37 \mathrm{~m}$ de carga, $8 \mathrm{~kW}$ de consumo de potencia, un $73 \%$ de rendimiento y un NPSH requerido en el orden de $4 \mathrm{~m}$.

Como se muestra en la Fig. 4, la máquina trabaja dentro del rango recomendado por el fabricante y marcado en amarillo, la característica de carga, con caudal entre 500 y $1200 \mathrm{l} / \mathrm{min}$, lo cual es totalmente satisfactorio y permite concluir que la selección del equipo es apropiada.

Dado que los problemas no se encuentran en la región de la descarga, el ruido y las vibraciones en los momentos en que la instalación deja de suministrar líquido hacen pensar en la existencia de cavitación durante el funcionamiento, lo cual es necesario estudiar.

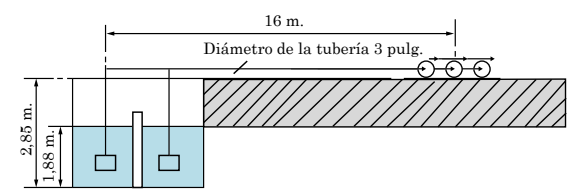

Fig. 5. Esquema del sistema de succión del equipo de bombeo del sistema hidroneumático.

$$
\text { Fuente: Autores. }
$$

Para la solución del problema técnico se analiza la presencia del fenómeno de la cavitación en el sistema, y para ello se determina la Carga Neta Positiva disponible en la Succión $\left(\mathrm{NPSH}_{\mathrm{d}}\right)$. Las dimensiones exteriores de la instalación en la región de succión están dadas en la Fig.5. La instalación posee una cisterna dividida por una pared interior, convirtiéndola en dos cisternas independientes. Cuando la bomba aspiraba de la cisterna más próxima no ocurría la falla, pero ambas cisternas no pueden ser conectadas debido a restricciones sanitarias.

Tabla 2. Datos PRincipales Del EQUipo de Bombeo.

\begin{tabular}{|c|c|}
\hline Tipo & CN 50-200 C \\
\hline $\mathrm{S} / \mathrm{N}$ & 0215S 15584-0001 \\
\hline $\mathrm{H}(\mathrm{m})$ & $46-20$ \\
\hline $\mathrm{H}_{\min }(\mathrm{m})$ & 20 \\
\hline $\mathrm{H}_{\max }(\mathrm{m})$ & 48,5 \\
\hline $\mathrm{Q}(1 / \mathrm{min})$ & $500-1200$ \\
\hline $\mathrm{P}(\mathrm{kW})$ & 9,2 \\
\hline $\mathrm{rpm}$ & 3400 \\
\hline $\mathrm{T}_{\max \text { fluido }}\left({ }^{\circ} \mathrm{C}\right)$ & 90 \\
\hline \multicolumn{2}{|c|}{ Fuente: Autores } \\
\hline
\end{tabular}

Las características técnicas del equipo de bombeo, así como los parámetros necesarios para la determinación del $\mathrm{NPSH}_{\mathrm{d}}$, son presentados en la tabla 2 .

En cualquier cálculo de fluidos por el interior de tuberías se hace necesario considerar el factor de fricción, factor que depende del material de la tubería, su diámetro nominal y el tipo de régimen de flujo (laminar o turbulento). Los datos del comportamiento del coeficiente de fricción de la tubería en régimen turbulento para un diámetro nominal de $65 \mathrm{~mm}$ son tomados del manual de Crane [15], donde el valor del coeficiente friccional $(\lambda \circ f t)$ es de 0,018 .

Los accesorios de tuberías presentes en la tubería de succión están dados en la Fig. 6. En ella se presenta el coeficiente de pérdidas del accesorio como una función del coeficiente de fricción de la tubería $(\lambda \circ f t)[15]$.

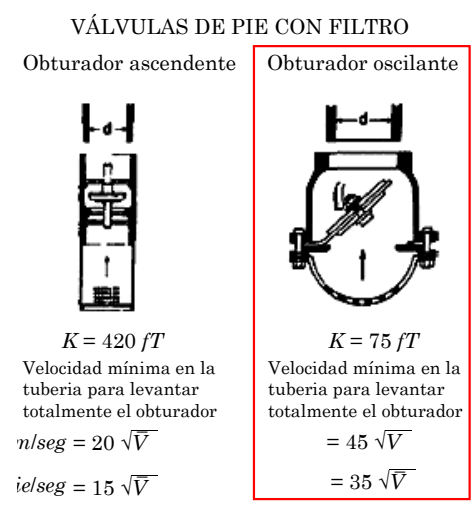

CODOS ESTÁNDAR

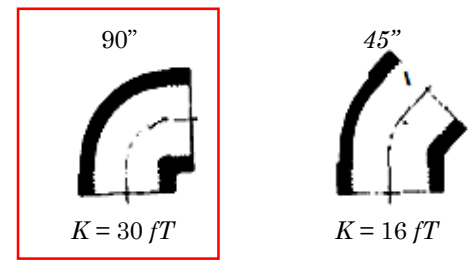

Fig. 6. Coeficiente de pérdidas del accesorio como una función del coeficiente de fricción de la tubería $(\lambda \circ \mathrm{ft})$. Fuente: [15].

Para este caso, el coeficiente de pérdida para el codo de $90^{\circ}$ es de 0,54 . Para la válvula de pie con obturador oscilante, el coeficiente de pérdida es de 1,35, y para la del obturador ascendente, el coeficiente de pérdida es de 7,56 .

Se realiza el cálculo del sistema en ambas condiciones al desconocer el tipo de válvula de pie existente en la instalación y no poder sacar de servicio el mismo.

Los valores de la presión de vapor $\left(\mathrm{P}_{v}\right)$ y peso específico $(\gamma)$ del agua en función de la temperatura están dados en la tabla 3 .

Tabla 3. Presión de Vapor $\left(\mathrm{P}_{V}\right)$ y peso específico $(\Gamma)$ DEL AGUA EN FUNCIÓN DE LA TEMPERATURA.

\begin{tabular}{|c|c|c|}
\hline $\mathrm{T}\left({ }^{\circ} \mathrm{C}\right)$ & $\mathrm{P}_{v}(\mathrm{~kg} / \mathrm{cm} 2)$ & $\gamma(\mathrm{kg} / \mathrm{dm} 3)$ \\
\hline 0 & 0,0062 & 0,9998 \\
\hline 10 & 0,0125 & 0,9996 \\
\hline 20 & 0,0238 & 0,9982 \\
\hline 30 & 0,0432 & 0,9955 \\
\hline \multicolumn{3}{|c|}{ Fuente: $[15]}$. \\
\hline
\end{tabular}


Para la determinación del $\mathrm{NPSH}_{d}$, se utiliza la ecuación 2 .

$$
\mathrm{NPSH}_{\mathrm{d}}=\frac{\mathrm{P}_{\mathrm{atm}}}{\rho \mathrm{g}}-\mathrm{Z}_{\mathrm{asp}}-\frac{\mathrm{V}_{2}^{2}}{2 \mathrm{~g}}-\sum_{1}^{2} \mathrm{hf}-\frac{\mathrm{P}_{\mathrm{vap}}}{\rho \mathrm{g}}
$$

Donde:

$P_{a t m}$ : Presión atmosférica $(\mathrm{Pa})$

$\rho: \quad$ Densidad del fluido $\left(\mathrm{kg} / \mathrm{m}^{3}\right)$

g: Aceleración de la gravedad $\left(\mathrm{m} / \mathrm{s}^{2}\right)$

$Z_{a s p}$ : Altura de aspiración (m)

$V: \quad$ Velocidad del fluido en la tubería $(\mathrm{m} / \mathrm{s})$

$\Sigma_{1}^{2} h f$ : Sumatoria de las pérdidas friccionales en la tubería $(\mathrm{m})$

$P_{v a p}$ : Presión de saturación a la temperatura del líquido $\left(\mathrm{P}_{\mathrm{a}}\right)$

Para tanques de grandes dimensiones, y considerando el nivel del líquido constante, se despreció la carga de velocidad $\left(\mathrm{V}_{2}^{2}\right) / 2 \mathrm{~g}$.

Trabajando en la ecuación 2 y aplicando la consideración anterior, se tiene la ecuación $2 \mathrm{a}$.

$$
\mathrm{NPSH}_{\mathrm{d}}=\frac{\mathrm{P}_{\mathrm{atm}}}{\rho \mathrm{g}}-\mathrm{Z}_{\mathrm{asp}}-\sum_{1}^{2} \mathrm{hf}-\frac{\mathrm{P}_{\mathrm{vap}}}{\rho \mathrm{g}}
$$

Las pérdidas friccionales se determinaron según la ecuación 3.

$$
\sum_{1}^{2} \mathrm{hf}=\lambda \frac{\mathrm{Lt}_{\mathrm{t}}}{\mathrm{d}} \frac{\mathrm{V}_{2}^{2}}{2 \mathrm{~g}}+\sum \xi \frac{\mathrm{V}_{2}^{2}}{2 \mathrm{~g}}
$$

Sustituyendo la velocidad (V) en función del caudal (Q) y los parámetros dados en la tabla 3, en la ecuación 2 a se obtiene la ecuación 4 .

Sustituyendo los valores, la ecuación 4 queda:

$\mathrm{NPSH}_{\mathrm{d}}=\frac{101325 \mathrm{~Pa}}{9810}-1,05 \mathrm{~m}-15904,13 \mathrm{Q}^{2}-\frac{4236,4}{9810}$

La tabla 4 presenta las magnitudes de los parámetros de la tubería y las propiedades termodinámicas del fluido de trabajo.

Tabla 4. Magnitudes de La tubería y PRopiedades TERMODINÁMICAS DE LA SUSTANCIA A USAR EN LA ECUACIÓN 2A.

\begin{tabular}{|c|c|}
\hline Parámetro & Magnitud \\
\hline Área de la tubería $\left(\mathrm{m}^{2}\right)$ & 0,0046 \\
\hline Altura de aspiración Z (asp) $(\mathrm{m})$ & 1,05 \\
\hline Longitud de la tubería $(\mathrm{m})$ & 18,92 \\
\hline Factor de fricción $\mathrm{A}$ & 0,018 \\
\hline K codo de 90 & 0,54 \\
\hline K válvula de pie (obturador \\
oscilante) & 1,35 \\
\hline K válvula de pie (obturador & 7,56 \\
\hline ascendente) & 4236,4 \\
\hline Pvapor $(\mathrm{Pa})$ & 1000 \\
\hline densidad $\left(\mathrm{kg} / \mathrm{m}^{3}\right)$ & 9,81 \\
\hline Aceleración de la gravedad $\left(\mathrm{m} / \mathrm{s}^{2}\right)$ & 101325 \\
\hline Patm (Pa) & 0,0762 \\
\hline diámetro $(\mathrm{m})$ & \\
\hline
\end{tabular}

Fuente: Autores.

\section{Resultados}

A partir de la expresión 4, en función del caudal (Q), fueron determinados los valores de $N P S H_{d} \min \mathrm{y}$ $\mathrm{NPSH}_{d}$ máx; considerando para ellos los valores de las dos válvulas que pueden ser empleadas, se presentaron los valores de $\mathrm{NPSH}_{\mathrm{r}}$ según el fabricante (Fig. 4). El valor del NPSHd min; $\mathrm{NPSH}_{\mathrm{r}}$ y $\mathrm{NPSH}_{\mathrm{d}}$ máx están dados en la tabla 5 .

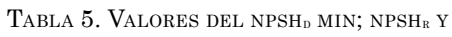
$\mathrm{NPSH}_{\mathrm{D}}$ MÁX EN FUNCIÓN DEL CAUDAL.

\begin{tabular}{|c|c|c|c|c|}
\hline $\begin{array}{c}\text { Caudal } \\
(\mathrm{l} / \mathrm{min})\end{array}$ & $\begin{array}{c}\text { Caudal } \\
\left(\mathrm{m}^{3} / \mathrm{s}\right)\end{array}$ & $\mathrm{NPSH}_{\mathrm{d}} \mathrm{min}$ & $\mathrm{NPSH}_{\mathrm{r}}$ & $\mathrm{NPSH}_{\mathrm{d}} \mathrm{max}$ \\
\hline 0 & 0 & 11,91 & 3,06 & 11,91 \\
\hline 200 & 0,0033 & 11,57 & 3,06 & 11,73 \\
\hline 300 & 0,0050 & 11,15 & 3,06 & 11,52 \\
\hline 400 & 0,0067 & 10,56 & 3,06 & 11,22 \\
\hline 500 & 0,0083 & 9,80 & 3,06 & 10,84 \\
\hline 600 & 0,0100 & 8,88 & 3,06 & 10,37 \\
\hline 700 & 0,0117 & 7,78 & 3,1 & 9,82 \\
\hline 800 & 0,0133 & 6,52 & 3,5 & 9,18 \\
\hline 900 & 0,0150 & 5,09 & 4 & 8,46 \\
\hline 1000 & 0,0167 & 3,50 & 5 & 7,65 \\
\hline 1100 & 0,0183 & 1,73 & 6 & 6,76 \\
\hline 1200 & 0,0200 & $-0,21$ & 8 & 5,78 \\
\hline
\end{tabular}

Fuente: Autores.

La representación gráfica de la tabla 5 está dada en la Fig. 7 y permite visualizar la relación entre los NPSH disponibles del sistema usando dos válvulas de pie diferentes y la comparación con el NPSH requerido.

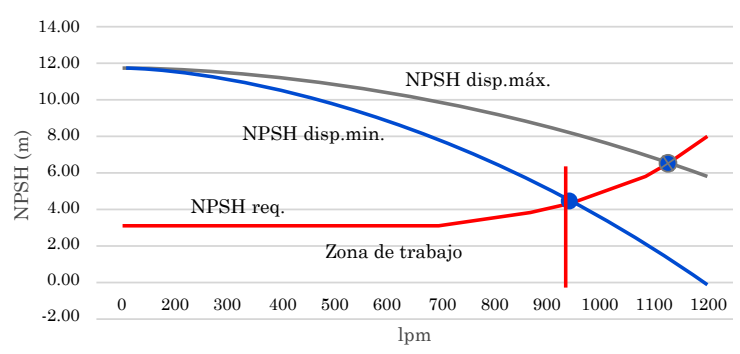

Fig. 7. Representación gráfica del NPSHr vs NPSHd para diferentes válvulas de pie en la succión de la tubería. Fuente: Autores.

En la Fig.7, se observa que el uso de diferentes válvulas de pie origina desiguales NPSH disponibles. El máximo dado para la válvula de pie articulada que ofrece menor resistencia hidráulica queda representado en gris en la Fig. 7. El mínimo dado con el uso de la válvula de pie ascendente de mayor resistencia hidráulica está representado en azul y ofrece un menor NPSHd. 
La intersección de ambas curvas, con la curva característica del NPSH requerido, ofrece dos puntos de funcionamiento. Para los autores, de acuerdo con los resultados obtenidos y las condiciones operacionales, la válvula usada es la de tipo ascendente y ello provoca que para caudales superiores a $900 \mathrm{l} / \mathrm{min}$, el punto de operación sea el menor, y la bomba, operada a caudales mayores de 900 l/min, trabaja en una región donde $\mathrm{NPSH}_{\mathrm{d}}<\mathrm{NPSH}_{\mathrm{r}}$, lo que provoca un estado de cavitación no totalmente desarrollada y pierde su capacidad de bombeo un tiempo después de la puesta en marcha.

Para la solución del problema, la dirección de mantenimiento de la institución hospitalaria sugirió tres variantes que fueron necesario analizar. La primera de ellas consiste en operar succionando desde la cisterna más cercana a los equipos de bombeo y trasvasar agua de la cisterna más alejada a la cisterna desde la que se succiona. Esto permitirá que, si el nivel es el adecuado dentro de la cisterna, la máquina no cavite. La Fig. 8, muestra la representación esquemática de la altura de succión donde debe de ocurrir la cavitación.

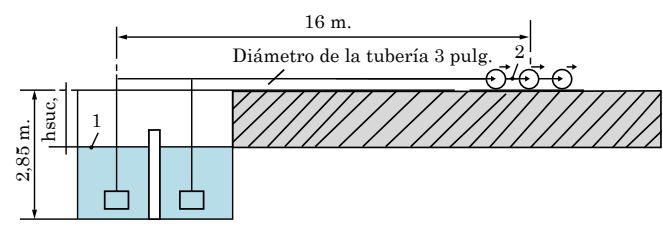

Fig. 8. Representación de la altura de succión donde debe ocurrir cavitación. Fuente: Autores.

Para el análisis de esta variante, se determina la altura de succión $\left(\mathrm{h}_{\mathrm{suc}}\right)$ del nivel del agua en la cisterna, a partir de la cual la máquina debe empezar a cavitar. Aplicando la ecuación 2 y tomando el $\mathrm{NPSH}_{\mathrm{d}}=$ $\mathrm{NPSH}_{\mathrm{r}}$ de la máquina, y sustituyendo la Zasp por el término $h_{\text {suc }}$, la ecuación 2 toma la representación dada en la ecuación 5 .

$$
\mathrm{NPSH}_{\mathrm{r}}=\frac{\mathrm{Patm}_{\mathrm{ag}}}{\rho \mathrm{g}}-\mathrm{h}_{\mathrm{suc}},-\frac{\mathrm{V}_{2}^{2}}{2 \mathrm{~g}}-\sum_{1}^{2} \mathrm{hf}-\frac{\mathrm{Pvap}_{\mathrm{va}}}{\rho \mathrm{g}}
$$

Por tanto,

$$
\begin{aligned}
& \mathrm{h}_{\text {suc }}=\frac{\mathrm{P}_{\text {atm }}}{\rho \mathrm{g}}-\sum_{1}^{2} \mathrm{hf}-\frac{\mathrm{P}_{\mathrm{vap}}}{\rho \mathrm{g}}-\mathrm{NPSH}_{\mathrm{r}} \\
& \mathrm{h}_{\mathrm{suc}}=1,38 \mathrm{~m}
\end{aligned}
$$

Por el cálculo anterior, la bomba debe comenzar a cavitar, si la válvula de pie es del tipo ascendente, a una profundidad de succión de 1,38 m. Ello resulta coincidente con las condiciones actuales de explotación del equipo.

Si la válvula fuera del tipo oscilante, al ser menor su pérdida friccional, la bomba podría succionar a una profundidad mayor, pero debe tenerse claro que las condiciones actuales de explotación no son las adecuadas.
La propuesta de la variante 2 se centra en sacar dos tomas de agua correspondiente a cada una de las cisternas y unir estas en un cabezal común, como se muestra en la vista superior de la instalación dada en la Fig. 9. La altura de salida de las tomas de agua debe estar a la altura del piso, como se muestra en la Fig. 10.

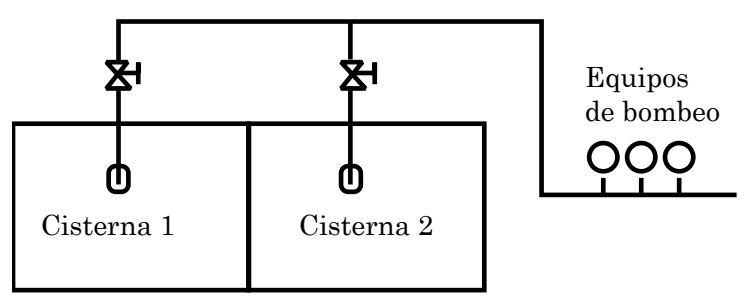

Fig. 9. Vista superior de la propuesta de la variante 2 . Fuente: Autores.

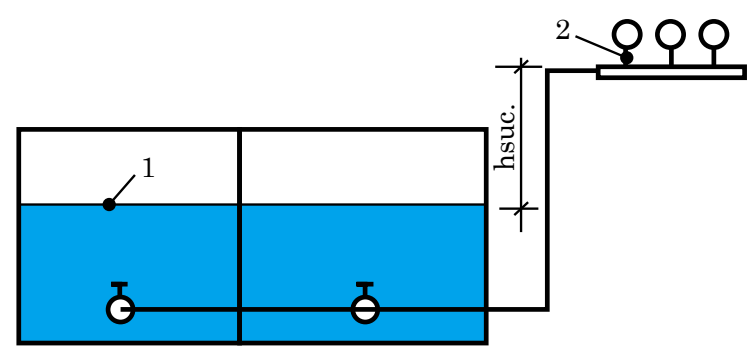

Fig. 10. Vista frontal de la propuesta de la variante 2 . Fuente: Autores.

En esta variante, igual que en la anterior, resulta necesario conocer la altura de succión a la que debe ocurrir la cavitación. Sustituyendo valores en la ecuación 5 presentada anteriormente, se tiene que la altura de succión $\left(\mathrm{h}_{\text {suc }}\right)$ es:

$$
\begin{aligned}
& h_{\text {suc }}=\frac{101325}{1000 * 9,81}-5,01-\frac{4236,4}{1000 * 9,81}-3,5 \\
& h_{\text {suc }}=10,32-5,01-0,43-3,5 \\
& h_{\text {suc }}=1,38 \mathrm{~m}
\end{aligned}
$$

Como se aprecia, no se logran cambios favorables con la modificación de la posición de la tubería por la parte exterior a la altura del piso. Ello está dado porque las longitudes de tuberías son las mismas y se adicionan accesorios de tuberías que aumentarían el valor de las pérdidas. No considerando estos, se ve que la altura de succión a la cual se iniciaría la cavitación corresponde a $1,38 \mathrm{~m}$.

La propuesta dada en la variante 3 es trasladar los equipos de bombeo y el hidropresor a una nueva posición con un nivel inferior al nivel de líquido en el interior de la cisterna, succión positiva (Fig. 11). La vista superior de esta variante queda presentada en la Fig. 12. 


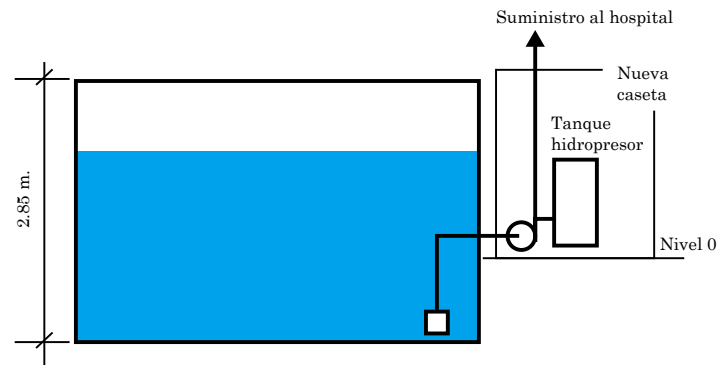

Fig. 11. Vista lateral de la variante 3 de solución. Fuente: Autores.

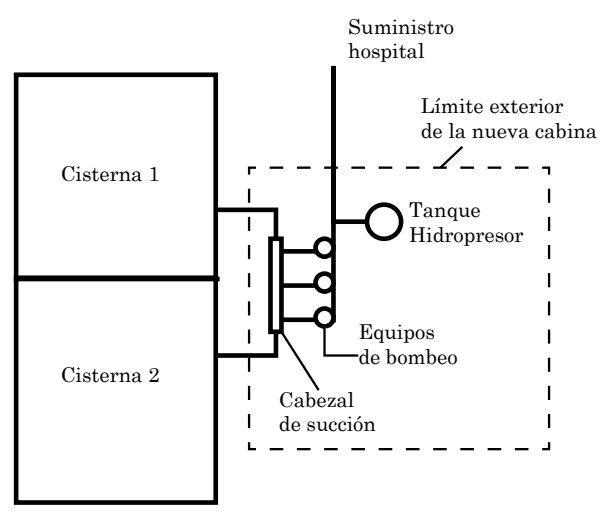

Fig. 12. Vista superior de la propuesta de la variante 3 . Fuente: Autores.

La variante 3 :

1. Permite succionar de cualquiera de las dos cisternas existentes sin peligro de contaminación si se toman las medidas establecidas.

2. No tiene peligro de cavitación en el equipo de bombeo dado que el sistema tiene succión positiva y, de colocar las válvulas de pie a las alturas normadas respecto al fondo el sistema, puede utilizar casi la totalidad del agua almacenada.

3. Al construir una nueva caseta de protección para el sistema de suministro de agua al hospital garantizará una mejor explotación del sistema y con menor ruido para operarios y vecinos de tomarse las medidas de protección acústicas normadas.

4. Tiene como inconveniente la necesidad de realizar una inversión para el traslado del equipamiento y construcción de la nueva caseta para el equipo hidropresor.

\section{Evaluación económica del} uso de los equipos de bombeo

El sistema en funcionamiento actual tiene un mayor consumo energético puesto que está obligado a operar las 24 horas para satisfacer los requerimientos de la institución hospitalaria. En la tabla 6 se presentan los consumos y costos energéticos anuales para ambos sistemas. La variante 1 se corresponde con el sistema hidroneumático y la variante 2 con el sistema actual.
Tabla 6. Cálculo del Consumo y costos energéticos anUales.

\begin{tabular}{|c|c|c|}
\hline Parámetros & $\begin{array}{c}\text { Variante } \\
1\end{array}$ & $\begin{array}{c}\text { Variante } \\
2\end{array}$ \\
\hline Potencia bomba (kW) & 8 & 6 \\
\hline $\begin{array}{c}\text { Horas de funcionamiento en el día } \\
\text { (h/día) }\end{array}$ & 15 & 24 \\
\hline Consumo energía (kWh/día) & 120 & 144 \\
\hline $\begin{array}{c}\text { Costo unitario de la energía } \\
\text { (\$/kWh) }\end{array}$ & 0,21 & 0,21 \\
\hline $\begin{array}{c}\text { Costo del consumo energético diario } \\
\text { (\$/día) }\end{array}$ & 25,2 & 30,24 \\
\hline $\begin{array}{c}\text { Costo anual (\$/año) } \\
\text { anual (kWh/año) }\end{array}$ & 9198 & 11037,60 \\
\hline $\begin{array}{c}\text { Diferencia en el consumo energético } \\
\text { Diferencia en el costo energético } \\
\text { anual (\$/año) }\end{array}$ & 8760,00 & \\
\hline
\end{tabular}

Fuente: Autores.

\section{Conclusiones}

La selección del sistema hidroneumático satisface los requerimientos del sistema de tubería, con la excepción de la solución dada en la región de succión que es la causa de la cavitación existente.

La determinación del NPSH disponible y su comparación con el NPSH requerido demuestra que para caudales superiores a $900 \mathrm{l} / \mathrm{min}$, la bomba trabaja en una región donde $\mathrm{NPSH}_{\mathrm{d}}<\mathrm{NPSH}_{\mathrm{r}}$, lo que provoca un estado de cavitación y, por tanto, la salida de funcionamiento del sistema.

En la variante 1 y 2 , propuesta por la dirección de mantenimiento de la institución, no se logran cambios favorables debido a que las longitudes de tuberías son las mismas y se adicionan accesorios de tuberías que aumentarían el valor de las pérdidas. Sin embargo, con la variante 3 , el sistema tiene succión positiva, y de colocar las válvulas de pie a las alturas normadas respecto al fondo el sistema puede utilizar casi la totalidad del agua almacenada sin presentar peligro alguno de cavitación.

La utilización del sistema auxiliar por la salida de servicio del sistema hidroneumático provoca gastos energéticos y económicos mayores para la institución hospitalaria. Las bombas actuales en

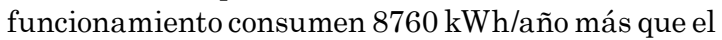
sistema hidroneumático. Con la utilización del sistema hidroneumático es posible ahorrar 1839 (\$/año).

\section{Financiamiento}

Artículo de investigación derivado del proyecto de investigación titulado "Gestión eficiente de la energía”, financiado por la Dirección Provincial de Salud Pública y la Universidad de Cienfuegos de Cuba. Fecha de inicio: 30 de agosto de 2017. Fecha de finalización: 26 de enero de 2018 . 


\section{Referencias}

[1] FIDE, Fascículo de FIDE de apoyo al programa de ahorro de energía del sector eléctrico, Recomendaciones para ahorro de energía en bombas centrífugas, México, mayo de 2005.

[2] L. E. Díaz, Y. Mustafá I y L. G. Rios, "Construcción y puesta en marcha de un banco de cavitación para bombas centrífugas pequeñas," Sci. Tech., vol. 1, no. 30, enero de 2006. http://dx.doi.org/10.22517/23447214.6499

[3] F. B. Cruz, "Análisis de las variables de la cavitación en bombas centrífugas horizontales", Tesis de pregrado, Instituto Politécnico Nacional, México, D. F., 2013.

[4] Z. Zou, F. Wang, Z. Yao, R. Tao, R. Xiao y H. Li, "Impeller radial force evolution in a large double-suction centrifugal pump during startup at the shut-off condition," Nucl. Eng. Des., vol. 310, pp. 410-417, diciembre de 2016. http:// dx.doi.org/10.1016/j.nucengdes.2016.10.034

[5] B. G. Park, Experimental study of debris head loss through a pressurized water reactor recirculation sump screen after LOCA, Nuclear Engineering and Design, vol. 241, no. 7, pp, 2462-2469, abril de 2011. http://dx.doi. org/10.1016/j.nucengdes.2011.04.013

[6] Z. Han y S. K. Vanapalli, "Relationship between resilient modulus and suction for compacted subgrade soils," Eng. Geol., vol. 211, pp. 85-97, agosto de 2016. http://dx.doi. org/10.1016/j.enggeo.2016.06.020

[7] P. Cao, Y. Wang, C. Kang, G. Li y X. Zhang, "Investigation of the role of non-uniform suction flow in the performance of water-jet pump," Ocean Eng., vol. 140, pp. 258-269, Aug. 2017. http://dx.doi.org/10.1016/j.oceaneng.2017.05.034

[8] T. Wu y J. D. Englehardt, "Mineralizing urban netzero water treatment: Field experience for energypositive water management," Water Res., vol. 106, pp. 352-363, diciembre de 2016. http://dx.doi.org/10.1016/j. watres.2016.10.015

[9] N. D. Karlsen-Davies y G. A. Aggidis, "Regenerative liquid ring pumps review and advances on design and performance," Appl. Energy, vol. 164, pp. 815-825, febrero de 2016. http://dx.doi.org/10.1016/j.apenergy.2015.12.041

[10] K. K. Botros, J. Geerligs y B. Watson, "Experimental investigation aiming at improving the suction flow capability of a gas expeller," J. Nat. Gas Sci. Eng., vol. 23, pp. 458-463, marzo de 2015. http://dx.doi.org/10.1016/j. jngse.2015.02.025

[11] J. Colt, P. Plesha y J. Huguenin, "Impact of net positive suction head on the design and operation of seawater pumping systems for use in aquaculture," Aquac. Eng., vol. 35, no. 3, pp. 239-257, octubre de 2006. http:// doi:10.1016/j.aquaeng.2006.03.001

[12] Y. Zhang, S. Hu, J. Wu, Y. Zhang y L. Chen, "Multi-objective optimization of double suction centrifugal pump using Kriging metamodels," Adv. Eng. Softw., vol. 74, pp. 16-26, agosto de 2014. http://dx.doi.org/10.1016/j.advengsoft.2014.04.001

[13] F. Burlon, D. Micheli, R. Furlanetto, M. Simonato y V. Cucit, "Cavitation Detection and Prevention in Professional Warewashing Machines," Energy Procedia, vol. 101, pp. 718-725, noviembre de 2016. https://doi.org/10.1016/j.egypro.2016.11.091

[14] G. Carraro, P. Pallis, A. D. Leontaritis, S. Karellas, P. Vourliotis, S. Rech y A. Lazzaretto, "Experimental performance evaluation of a multi-diaphragm pump of a micro-ORC system," Energy Procedia, vol. 129, pp. 1018-1025, septiembre de 2017. https://doi.org/10.1016/j. egypro.2017.09.232

[15] CRANE, Fluid flow: in valves, accessories and pipes, McGraw-Hill Interamericana, 2011.

[16] Technical Data CN50-200C, Sea Land, Cod: 175128000, (PD) Italia, Disponible en: www.sea-land.it
José P. Monteagudo Yanes. Ingeniero mecánico de la Universidad Central de Las Villas (UCLV), Cuba, 1975. Doctor en Ciencias Técnicas del Instituto Superior Politécnico “José A. Echeverría”, La Habana, 1997. Profesor titular y profesor consultante de la Universidad de Cienfuegos. Es miembro del consejo científico, del comité académico de la maestría en Eficiencia energética y del comité académico del doctorado de la Facultad de Ingeniería de la Universidad de Cienfuegos. Es miembro del comité de expertos del Ministerio de Ciencia, Tecnología y Medio Ambiente y miembro del Tribunal Nacional para la Defensa de Grados Científicos de la República de Cuba en la rama energética mecánica. https://orcid.org/00000002-7494-2477

Reinier Jiménez Borges. Recibió el título de Ingeniero mecánico en el 2014. Obtuvo el título de magíster en Eficiencia energética en el año 2017 de la Universidad de Cienfuegos. Hasta la fecha se ha desempeñado como profesor de Mecánica de los Fluidos. Ha publicado varios artículos en revistas nacionales e internacionales. Actualmente, es miembro del grupo de investigación sobre Eficiencia energética y fuentes renovables de energía. https://orcid.org/00000001-6451-8499 